\title{
Recent advances in the diagnosis and manage- ment of interstitial pneumonia with autoimmune features: the perspective of rheumatologists
}

\author{
Hong Ki Min ${ }^{1}$, Se-Hee Kim¹, Sang-Heon Lee ${ }^{2}$, and Hae-Rim Kim²
}

\begin{abstract}
${ }^{1}$ Division of Rheumatology, Department of Internal Medicine, Konkuk University Medical Center, Seoul; ${ }^{2}$ Division of Rheumatology, Department of Internal Medicine, Research Institute of Medical Science, Konkuk University School of Medicine, Seoul, Korea
\end{abstract}

\section{Received: August 21, 2020 \\ Accepted: October 7, 2020}

\section{Correspondence to}

Hae-Rim Kim, M.D.

Department of Rheumatology,

Konkuk University Medical

Center, 120-1 Neungdong-ro,

Gwangjin-gu, Seoul 05030,

Korea

Tel: +82-2-2030-7542

Fax: +82-2-2030-7728

E-mail: kimhaerim@kuh.ac.kr

https://orcid.org/0000-0002-

$1911-6236$
Interstitial pneumonia with autoimmune feature (IPAF) is a recently established disease entity that is comprised of interstitial lung diseases with evidence of autoimmune features but that does not fulfill the criteria for definite autoimmune rheumatic diseases. The classification criteria for IPAF were defined by the European Respiratory Society and American Thoracic Society in 2015. However, further studies to establish IPAF subgroups and treatment modalities for each subgroup are still needed. In this review, we discuss recent advances regarding IPAF and raise critical points for the diagnosis and management of patients with IPAF from the perspective of rheumatologists.

Keywords: Lung diseases, interstitial; Autoimmune diseases; Rheumatology; Therapy; Prognosis

\section{INTRODUCTION}

Interstitial lung disease (ILD) is a group of lung diseases that affect the interstitium with inflammatory and fibrotic insults. ILD is divided into four different categories according to etiology as follows: (1) occupational/ environmental factors; (2) iatrogenic factors; (3) factors associated with connective tissue diseases (CTDs; as in autoimmune rheumatic disease [ARD]); and (4) idiopathic factors [1]. The concept of ILD with undifferentiated CTD was first proposed by Kinder et al. [2]. Thereafter, the European Respiratory Society (ERS) and American Thoracic Society (ATS) Task Force on Undifferentiated
Forms of CTD-associated ILD designated this disease as interstitial pneumonia with autoimmune feature (IPAF) and established the classification criteria for IPAF [3]. A certain portion of previously diagnosed idiopathic ILD cases can now be reclassified as IPAF. However, the current classification criteria for IPAF have several limitations as they do not include critical clinical domain items and the heterogeneity of IPAF cases has been ignored. Here, we review recent advances in the diagnosis and management of IPAF and propose critical points regarding IPAF from the perspective of rheumatologists. 


\section{DEFINITION AND CLASSIFICATION CRITERIA FOR IPAF}

Cases of currently defined IPAF were formerly classified as idiopathic ILD. Among idiopathic ILDs, some cases show autoimmune traces, which have been referred to, using various terms, as follows: "undifferentiated CTD-associated ILD," "lung-dominant CTD," or "autoimmune-featured ILD" [2,4-6]. The former nomenclatures have similarities with regard to distinguishing ILD with autoimmune traces; however, the detailed classification criteria differ from each other. The ERS/ ATS Task Force redefined and unified the former nomenclature and newly established classification criteria for IPAF [3]. The fundamental entry criteria for IPAF are as follows: (1) presence of interstitial pneumonia; (2) exclusion of other possible etiologies of ILD; and (3) not fulfilling the definite criteria for ARD [3]. Furthermore, cases that satisfy the entry criteria should fulfill at least two of the following domains of the IPAF classification criteria: (1) clinical domain; (2) serological domain; and (3) morphological domain (Table 1). The usual interstitial pneumonia (UIP) pattern does not satisfy the morphological domain of the classification criteria; however, if the UIP pattern fulfills the other two domains (clinical and serological domains), it can be classified as IPAF. The classification criteria for IPAF require a multidisciplinary approach that involves pulmonologists, rheumatologists, radiologists, laboratory physicians, and pathologists.

\section{EPIDEMIOLOGY AND PREVALENCE OF THE CLINICAL, SEROLOGICAL, AND MORPHOLOG- ICAL FINDINGS OF IPAF}

The prevalence of IPAF ranges from $7.1 \%$ to $34.1 \%$ of all ILDs [7-10], and the prevalence is varied because all studies reported to date have been conducted retrospectively. The average age of patients with IPAF is in the mid-6os, and most studies have shown a female predominance [9-15], whereas some have suggested an equal sex distribution $[7,8]$. IPAF showed a clear difference from idiopathic pulmonary fibrosis (IPF), which shows male predominance. The reported prevalence of former or current smoking history in patients with IPAF ranges from $38.8 \%$ to $56.2 \%[7,11,16,17]$. The most common morphological pattern is non-specific interstitial pneumonia (NSIP) in IPAF, with a prevalence of $42.1 \%$ to $68.9 \%$ [7,8,10,11,13-15,17,18]. However, some reports showed UIP dominance in IPAF, which was found in $27.9 \%$ to $44.2 \%$ of cases $[12,16,19,20]$.

Among the three domains of the IPAF classification criteria, the morphological domain is the most frequently fulfilled domain (78.9\% to $100 \%$ ), followed by the serological and clinical domains accounting for $48.9 \%$ to $93.0 \%$ and $31.5 \%$ to $62.2 \%$ of cases, respectively $[7-10,15,17,21]$. The most common findings in the clinical domain are Raynaud's phenomenon (8.89\% to $74.1 \%)$, mechanic's hands ( $4 \%$ to $29 \%$ ), inflammatory arthritis/ morning stiffness ( $16 \%$ to $76.5 \%$ ), and Gottron's sign (5\% to $18 \%)[7-10,15-17,19]$. In the serological domain, antinuclear antibody (ANA) titers $\geq 1: 320$ in diffuse, speckled, homogeneous patterns or any titer with a nucleolar or centromere pattern are the most frequent findings (10.3\% to $82.4 \%$ ), followed by rheumatoid factor ( $4.4 \%$ to $28.6 \%$ ), anti-Ro ( $4.4 \%$ to $55.9 \%)$, and anti-tRNA synthetase positivity (o\% to $35 \cdot 7 \%$ ) [7-10,15-17,19].

\section{COMPARISON BETWEEN IPAF AND ARD-ILD}

ILD is a relatively common pulmonary manifestation in various ARDs, such as systemic sclerosis (SSc), inflammatory myositis, primary Sjögren's syndrome (pSS), and rheumatoid arthritis (RA). The highest incidence of ILD in ARDs is found in patients with SSc (43.0\% to 91.0\%) [22-24], followed by those with inflammatory myositis (19.9\% to $78 \%$ ) [25-27], RA (7.7\% to $33.0 \%$ ) [28,29], and pSS (8.0\% to $39.1 \%$ ) [30-32]. The positive findings of the clinical and serological domains of the IPAF groups demonstrate that the features associated with SSc, RA, inflammatory myositis, and pSS are frequently found in patients with IPAF. Although most features of the clinical and serological domains show similar prevalence rates with ARD-associated ILD (ARD-ILD, formerly CTD-ILD), the SSc-specific autoantibody, anti-topoisomerase (Scl-70), is not predominantly present in the IPAF group (o\% to $5.7 \%$ ) [7-10,15,17,19]. However, in the serological domain, ANA positivity in the IPAF classification criteria includes any titer with a nucleolar or centromere pattern, which is the typical pattern of 
Table 1. Classification criteria of interstitial pneumonia with autoimmune features defined by ERS/ATS

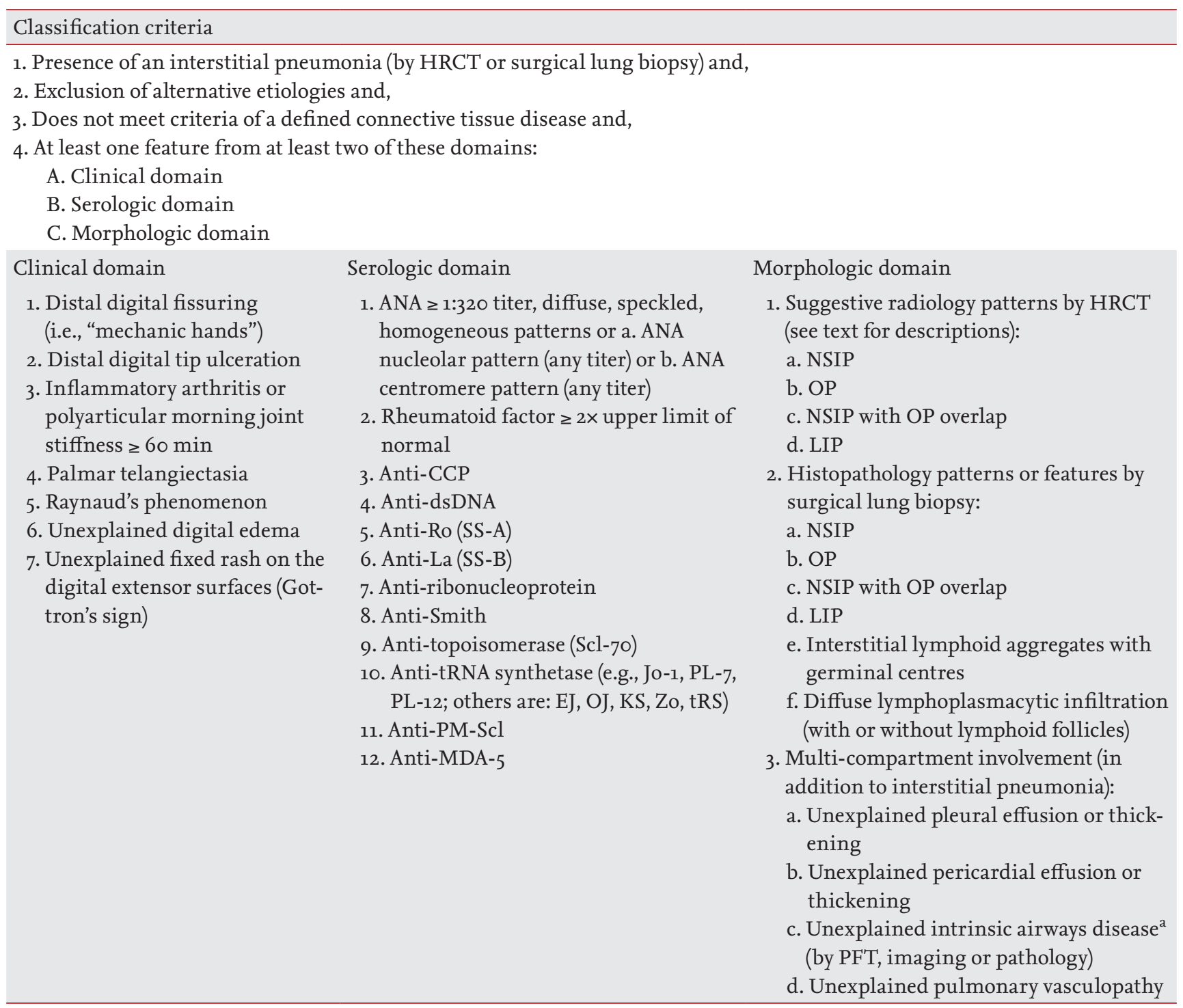

HRCT, high-resolution computed tomography; ANA, antinuclear antibody; CCP, cycli citrullinated peptide; dsDNA, doublestranded DNA; tRNA, transfer tRNA, transfer ribonucleic acid; PL-7, threonyl-tRNA synthetase; EJ, glycyl-tRNA synthetase; OJ, isoleucyl-tRNA synthetase; KS, asparaginyl-tRNA synthetase; Zo, phenylalanyl-tRNA synthetase; tRS, threonyl-tRNA synthetase; PM, polymyositis; MDA-5, melanoma differentiation-associated gene 5 ; NSIP, non-specific interstitial pneumonia; OP, organizing pneumonia; LIP, lymphoid interstitial pneumonia; PFT, pulmonary function test.

${ }^{a}$ Intrinsic airway disease includes airflow obstruction, bronchiolitis, or bronchiectasis.

limited cutaneous SSc [33]. In patients with SSc, anti-topoisomerase antibody positivity is more closely associated with the presentation of ILD [34,35], and this characteristic differs from that observed in the IPAF cohort. This discordance may be due to the difference between IPAF and ARD-ILD.

\section{PATHOPHYSIOLOGY AND GENETIC BACK- GROUND OF IPAF}

The pathophysiology of IPAF has not been fully elucidated. As included in the nomenclature for IPAF, patients with IPAF are assumed to have features of both idiopathic interstitial pneumonia (IIP) and ARD-ILD, which are represented by idiopathic fibrosis and the 
autoimmune-mediated inflammatory process (Fig. 1). Several genetic polymorphisms are known to be involved in the pathogenesis of ILD [36,37]. IPF-associated gene mutations are found in patients with telomerase-, surfactant protein-, immune function-, and mucin ${ }_{5} \mathrm{~B}\left(\mathrm{MUC}_{5} \mathrm{~B}\right)$-related conditions [37]. Newton et al. [38] demonstrated that the leukocyte telomere length is longer in patients with IPAF and ARD-ILD than in those with IPF. The leukocyte telomere length is associated with pulmonary function and lung transplant outcome in patients with IPAF [38]. Furthermore, $M U_{5} B$ polymorphism is more common in patients with IPAF than in those with IPF, but the same is not true for Toll-interacting protein (TOLLIP) polymorphism [38]. These findings confirm that IPAF and IPF have similar ge-

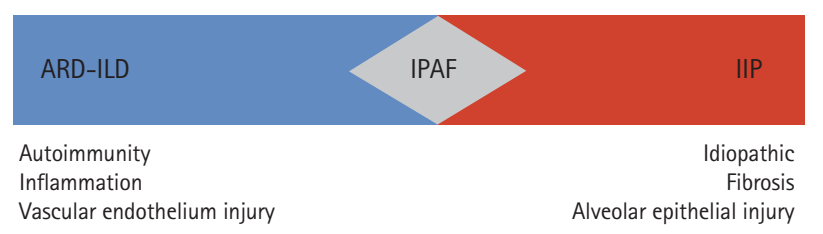

Figure 1. Schematic spectra of interstitial pneumonia with autoimmune feature (IPAF), idiopathic interstitial pneumonia (IIP), and autoimmune rheumatic disease-associated interstitial lung disease (ARD-ILD) and the representative features of IIP and ARD-ILD. netic backgrounds in terms of pathogenesis but show different detailed genetic mutations. Numerous genetic polymorphisms are found in ARDs, such as RA, pSS, and SSc, but there have been no studies regarding the association between ARD-related genes and the development of IPAF. Therefore, a genome-wide association study in patients with IPAF could provide new insights into the pathogenesis of IPAF.

Another issue is the influence of environmental factors on the pathophysiology of IPAF. As mentioned in the previous section, history of smoking is associated with the development of IPAF. In the field of rheumatology, smoking has been shown to play a role in disease progression and even in autoantibody formation. Anti-citrullinated protein antibody positivity is associated with history of smoking in RA [39,40], and former smokers have higher odds ratios for anti-synthetase antibody positivity in inflammatory myositis [41]. Among patients with pSS, those with a history of smoking (current and former smokers) had a higher ANA positivity rate than never smoker [42]. In an animal model of RA, smoking was shown to enhance peptide citrullination in lung tissue and tracheal cartilage [43]. This suggested that smoking can locally promote anti-citrullinated peptide antibody production, especially in the lungs. Although

Table 2. Suggested subclassification of interstitial pneumonia with autoimmune features

\begin{tabular}{|c|c|c|c|c|}
\hline & SSc type & Arthritis type & Myositis type & SLE/pSS type \\
\hline Clinical domain & $\begin{array}{l}\text { 1. Digital tip ulcer } \\
\text { 2. Palmar telangiectasia } \\
\text { 3. Raynaud's phenomenon } \\
\text { 4. Unexplained digital edema }\end{array}$ & $\begin{array}{l}\text { 1. Inflammatory } \\
\text { arthritis or } \\
\text { polyarticular } \\
\text { morning joint } \\
\text { stiffness } \geq 60 \text { min }\end{array}$ & $\begin{array}{l}\text { 1. Distal digital } \\
\text { fissuring (i.e., } \\
\text { "mechanic hands") } \\
\text { 2. Unexplained fixed } \\
\text { rash on the digital } \\
\text { extensor surfaces } \\
\text { (Gottron's sign) }\end{array}$ & \\
\hline Serologic domain & $\begin{array}{l}\text { 1. ANA nucleolar pattern } \\
\text { (any titer) or ANA } \\
\text { centromere pattern } \\
\text { (any titer) } \\
\text { 2. Anti-topoisomerase (Scl-7o) } \\
\text { 3. Anti-ribonucleoprotein } \\
\text { 4. Anti-PM-Scl }\end{array}$ & $\begin{array}{l}\text { 1. Rheumatoid factor } \\
\geq 2 \times \text { upper limit of } \\
\text { normal } \\
\text { 2. Anti-CCP }\end{array}$ & $\begin{array}{l}\text { 1. Anti-tRNA } \\
\text { synthetase } \\
\text { (e.g., Jo-1, PL-7, PL- } \\
\text { 12; others are: EJ, OJ, } \\
\text { KS, Zo, tRS) } \\
\text { 2. Anti-MDA-5 }\end{array}$ & $\begin{array}{l}\text { 1. ANA } \geq 1: 320 \text { titer, } \\
\text { diffuse, speckled, } \\
\text { homogeneous patterns } \\
\text { 2. Anti-dsDNA } \\
\text { 3. Anti-Ro (SS-A) } \\
\text { 4. Anti-La (SS-B) } \\
\text { 5. Anti-Smith }\end{array}$ \\
\hline
\end{tabular}

SSc, systemic sclerosis; SLE, systemic lupus erythematosus; pSS, primary Sjögren's syndrome; ANA, antinuclear antibody; PM, polymyositis; CCP, cycli citrullinated peptide; tRNA, transfer RNA; tRNA, transfer ribonucleic acid; PL-7, threonyl-tRNA synthetase; EJ, glycyl-tRNA synthetase; OJ, isoleucyl-tRNA synthetase; KS, asparaginyl-tRNA synthetase; Zo, phenylalanyltRNA synthetase; tRS, threonyl-tRNA synthetase; MDA-5, melanoma differentiation-associated gene 5; dsDNA, doublestranded DNA. 
smoking is associated with the development of ILD, it could affect the development of IPAF via autoantibody formation.

\section{SUBCLASSIFICATION OF IPAF}

In the classification criteria for IPAF, the items that constitute the clinical and serological domains are derived from the features of specific ARDs. Therefore, it is possible to divide IPAF into several subtypes according to the items of the clinical and serological domains (Table 2). Subgrouping IPAF according to the following four categories, (1) SSc type, (2) arthritis type, (3) myositis type, and (4) systemic lupus erythematosus (SLE)/pSS type, may be considered in future revision of the classification criteria for IPAF. We suggest these IPAF subgroups in the present review because although ARDs have definite similarity with regard to autoimmune-mediated processes, they vary in their detailed pathogenesis, clinical features, and serological markers. Furthermore, subgrouping IPAF could provide insights and concepts for establishing individualized treatment strategies. The 2015 classification criteria include various patients with IPAF but have significant limitations in that they ignore the specific features of each ARD and classify too many heterogenic patients into a single IPAF category.

\section{POTENTIAL NOVEL DIAGNOSTIC TOOL FOR IPAF}

The 2015 ERS/ATS classification criteria for IPAF are significant as they attempt to establish IPAF as a novel disease entity and create a basis for conducting future research on IPAF. However, updating the clinical domains with more objective signs could improve the value of the classification criteria. The inclusion of inflammatory arthritis may increase the validity of the criteria using imaging studies, such as ultrasonography, and limit the involvement of small joints, which are mainly involved in RA [44]. In the clinical domain associated with SSc, adding abnormal nailfold capillaroscopic findings, which are among the classification criteria for SSc, could be considered [45]. Two research studies reported abnormal nailfold capillaroscopic findings in approximately $20 \%$ of patients with IPAF $[8,10]$. Tirelli et al. [18] suggested that a multidisciplinary approach including nailfold capillaroscopy could improve the differentiation between IPAF, ARD-ILD, and idiopathic ILD. Several parameters can be observed in nailfold capillaroscopy for patients with SSc, including (1) irregularly enlarged capillaries, (2) giant capillaries, (3) hemorrhage, (4) loss of capillaries (avascular area), (5) disorganization of the vascular array, and (6) capillary ramifications [46]. In our clinic, we performed nailfold capillaroscopy examinations in patients with IPAF and found abnormalities (Fig. 2). Although the prevalence of ILD is relatively high
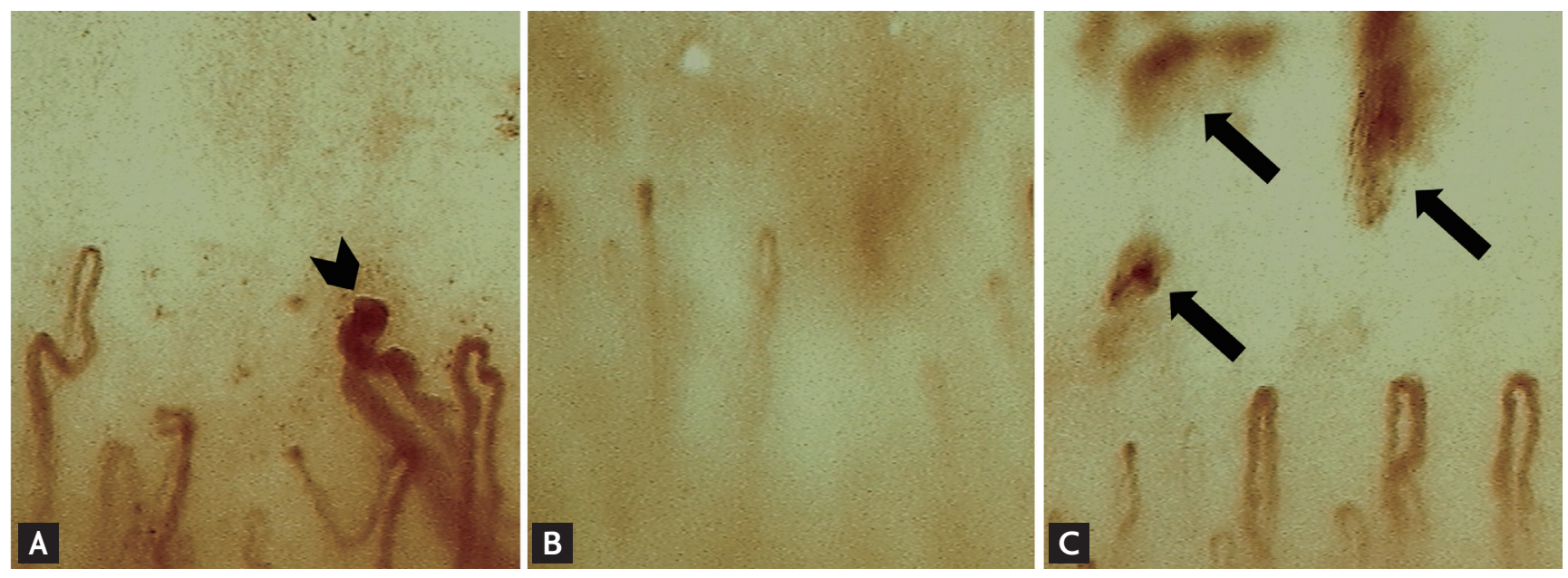

Figure 2. Abnormal nailfold capillaroscopy findings in a 52-year-old female patient with interstitial pneumonia with autoimmune features with a rheumatoid factor level of $45 \mathrm{IU} / \mathrm{mL}$ (reference range, o to 18) and an non-specific interstitial pneumonia-dominant morphological pattern. (A) Giant capillary (black arrowhead). (B) Avascular area. (C) Hemorrhage (black arrows). 
in pSS, the 2015 classification criteria for IPAF do not include clinical domains related to pSS. The revised 2016 classification criteria for pSS include objective signs of dry eyes and mouth by Shirmer's test and unstimulated whole saliva flow rate [47]. These are noninvasive and convenient means of checking objective signs of sicca symptoms. The recently revised classification criteria for SLE were updated with a scoring system [48]. High scores are assigned to items on cutaneous lupus symptoms, serositis (pleural and pericardial effusions and pericarditis), and proteinuria [48]. These items could be evaluated by inspection (cutaneous lupus), radiography (serositis), and quantitative urine analysis (proteinuria). In the myositis-associated clinical domains, the most typical clinical presentation and signs of inflammatory myositis are proximal muscle weakness and elevated levels of muscle enzymes (creatinine kinase, lactate dehydrogenase, aldolase, aspartate aminotransferase, and alanine aminotransferase) [49]. Therefore, the typical proximal muscle weakness and elevated levels of muscle enzymes should be considered potential clinical domains of the classification criteria for IPAF in future. Anti-neutrophil cytoplasmic antibody (ANCA)-associated vasculitis (AAV) is a systemic vasculitis mainly involving the small vasculature, and myeloperoxidase (MPO)-ANCA and proteinase 3 ( $\left.\mathrm{PR}_{3}\right)$-ANCA are the most common autoantibodies found in AAV. The prevalence of ILD ranged from $46 \%$ to $71 \%$ among patients with MPO-ANCA-positive AAV and from o\% to $29 \%$ in those with $\mathrm{PR}_{3}$-ANCA-positive AAV [50]. In future, the revised classification criteria for IPAF may be updated by including clinical and serological domains to enhance the usefulness of the criteria by considering the objective signs and examinations used in the recent classification criteria for ARDs.

\section{TREATMENT OF IPAF}

The management of IPAF is based on clinical trials of ARD-ILD and IIP due to the lack of controlled interventional clinical trials in patients with IPAF. Usually, immunosuppressants, including systemic glucocorticoid and additional antifibrotic agents, are used for patients with IPAF. The fundamental hypothesis for the use of immunosuppressants in ARD-ILD is that immune and inflammatory reactions are the causes of ILD progression in patients with ARD. Furthermore, alveolar epithelial injury, which is the main pathological process in IIP, is also assumed to be a critical factor in the development of ARD-ILD [51]. Initial glucocorticoid therapy with the addition of glucocorticoid-sparing agents, such as mycophenolate mofetil, azathioprine, cyclophosphamide, and calcineurin inhibitors, is the main treatment modality for ARD-ILD [52]. However, the use of glucocorticoids and immunosuppressants did not significantly reduce the hazard ratio (HR) of mortality in IPAF [7,8,12,14], but Li et al. [53] reported that patients with IPAF who received immunosuppressant therapy showed improvement of the diffusion capacity of the lungs for carbon monoxide $\left(\mathrm{DL}_{\mathrm{CO}}\right)$ based on 6-month follow-up data.

Recently, the antifibrotic agent pirfenidone has been shown to be effective in improving the pulmonary function and survival of patients with IPF [54-56]. Another antifibrotic agent, nintedanib, is a tyrosine kinase inhibitor initially developed as an antitumor agent that suppresses the vascular endothelial growth factor pathway. Nintedanib significantly reduces the decline in forced vital capacity (FVC) and mortality rate in patients with IPF [57,58]. Pirfenidone therapy for SSc-ILD failed to show any beneficial effects on FVC and other clinical parameters (dyspnea score, 6-minute walking distance, and modified Rodnan skin score) [59]. In contrast, nintedanib use in SSc-ILD showed better prognosis than placebo in terms of decline in FVC [6o]. In studies on IPAF, antifibrotic agents did not show any beneficial effects in reducing mortality $[8,9]$. The most important limitation in establishing a treatment strategy for IPAF is that no randomized clinical trials of immunosuppressant and antifibrotic agents for IPAF have yet been conducted. A number of clinical trials have been designed to confirm the therapeutic effects of antifibrotic agents in unclassified ILD, including IPAF [61,62], and these may provide more evidence to support the use of antifibrotic agents in IPAF. The medications used in IPAF patients in previous studies are summarized in Table 3.

\section{PROGRESSION OF IPAF TO ARD-ILD}

The progression of IPAF to definite ARD is an interest- 
Table 3. Medications used in patients with interstitial pneumonia with autoimmune features

\begin{tabular}{|c|c|c|c|}
\hline \multirow{2}{*}{$\begin{array}{l}\text { Study } \\
\text { Chartrand et al. }(2016)[15](n=56)\end{array}$} & \multicolumn{2}{|l|}{ Medication } & \multirow{2}{*}{$\begin{array}{ll} & \text { Outcome (HR for mortality) } \\
\text { NA } & \end{array}$} \\
\hline & Prednisone & $45(81.8 \%)$ & \\
\hline & Mycophenolate mofetil & $42(76.4 \%)$ & \\
\hline & Azathioprine & $20(36.4 \%)$ & \\
\hline & Cyclophosphamide & $13(23.6 \%)$ & \\
\hline & Tacrolimus & $4(7 \cdot 3 \%)$ & \\
\hline & Rituximab & $2(3.6 \%)$ & \\
\hline \multirow[t]{5}{*}{ Oldham et al. (2016) [7] $(n=144)$} & Glucocorticoid & $46(32.2 \%)$ & Glucocorticoid: HR, 1.39 \\
\hline & Azathioprine & $41(28.5 \%)$ & $\left(95 \% \mathrm{CI}, 0.73^{-2.63)}\right.$ \\
\hline & Mycophenolate mofetil & $19(13.2 \%)$ & IS: HR, 0.70 (95\% CI, 0.35-1.40) \\
\hline & Tacrolimus & $4(2.8 \%)$ & \\
\hline & Cyclophosphamide & $2(1.4 \%)$ & \\
\hline \multirow[t]{3}{*}{ Ahmad et al. (2017) [8] $(n=57)$} & Glucocorticoid & $38(67.9 \%)$ & NA \\
\hline & Immunosuppressant & $16(28.6 \%)$ & \\
\hline & Antifibrotic agent & $3(5.4 \%)$ & \\
\hline \multirow[t]{4}{*}{ Ito et al. $(2017)[11](\mathrm{n}=98)$} & Glucocorticoid & $17(17 \cdot 3)$ & NA \\
\hline & Glucocorticoid + IS & $48(49.0 \%)$ & \\
\hline & Glucocorticoid + IS + pirfenidone & $1(1.0 \%)$ & \\
\hline & Pirfenidone & $2(2.0 \%)$ & \\
\hline \multirow[t]{3}{*}{ Dai et al. $(2018)[14](n=177)$} & Glucocorticoid & $88(49.7 \%)$ & Glucocorticoid: HR, 0.655 \\
\hline & IS & $4(2.3 \%)$ & $\begin{array}{l}\text { (95\% CI, } 0.329-1.304) \\
\text { IS: HR, } 0.786 \text { (95\% CI o.325-1.902) }\end{array}$ \\
\hline & Glucocorticoid + IS & $40(22.6 \%)$ & \\
\hline \multirow[t]{3}{*}{ Yoshimura et al. $(2018)[17](n=32)$} & Glucocorticoid & $19(59.4 \%)$ & NA \\
\hline & IS & $11(34.4 \%)$ & \\
\hline & Pirfenidone & $8(25.0 \%)$ & \\
\hline \multirow[t]{2}{*}{ Kim et al. $(2020)[12](\mathrm{n}=109)$} & Glucocorticoid & $22(20.2 \%)$ & $\begin{array}{l}\text { Glucocorticoid: HR, } 1.100 \\
(95 \% \text { CI, o.565-2.142) } \\
\text { Glucocorticoid + IS: HR, } 0.768 \\
(95 \% \text { CI, o.438-1.344) }\end{array}$ \\
\hline & Glucocorticoid + IS & $70(64.2 \%)$ & \\
\hline \multirow[t]{5}{*}{ Sebastiani et al. $(2020)[16](n=52)$} & Glucocorticoid & $33(63.5 \%)$ & NA \\
\hline & Mycophenolate mofetil & $5(9.6 \%)$ & \\
\hline & Azathioprine & $5(9.6 \%)$ & \\
\hline & Cyclophosphamide & $4(7 \cdot 7 \%)$ & \\
\hline & Antifibrotic agent & $6(11.5 \%)$ & \\
\hline
\end{tabular}

HR, hazard ratio; NA, not applicable; CI, confidence interval; IS, immunosuppressant.

ing issue. As shown in Fig. 1, IPAF is assumed to have the characteristics of both IIP and ARD-ILD. Four studies reported the progression rates of IPAF to definite ARD and showed that $12.2 \%, 13.5 \%, 16 \%$, and $18.8 \%$ of
IPAF cases had progressed to ARD at 4.5, 2.6, 1, and 5.2 years of follow-up, respectively $[11,16,21,63]$. Combining the results of the four studies, we found RA in 11 cases, SSc in six, pSS in five, polymyositis in five, AAV in two, 
overlap syndrome in three, and SLE in one $[11,16,21,63]$. In the field of rheumatology, the concept of "preclinical ARD" has recently been settled, and close follow-up with early treatment has been discussed $[64,65]$. In one study, 6 months of treatment with immunosuppressants improved the $\mathrm{DL}_{\mathrm{CO}}$ significantly in patients with IPAF, supporting the usefulness of early treatment in IPAF [53]. In one study, 14\% of RA patients showed ILD prior to arthritis symptoms [66]. Furthermore, ARD patients presenting ILD as the first manifestation can be classified as IPAF. Therefore, assuming IPAF to be a preclinical ARD and starting early treatment should be considered in patients with IPAF.

\section{PROGNOSIS OF PATIENTS WITH IPAF}

The prognosis of patients with ILD focuses on the decline of pulmonary function, acute exacerbation (AE) events, and survival rate [67-69]. Pulmonary functions assessed using the $\mathrm{DL}_{\mathrm{CO}}$ and total lung capacity progress more slowly in patients with IPAF than in those with IIP $(-1.21 \%$ vs. $-4.58 \%$ and $-0.75 \%$ vs. $-2.32 \%$ predicted/ year, respectively), and these are similar when compared between patients with IPAF and ARD-ILD [12]. Collins et al. [20] demonstrated that the $\mathrm{DL}_{\mathrm{CO}}$ had improved in patients with IPAF but declined in those with IPF or ARD-ILD, and FVC had declined in all the patients at 1-year follow-up. Another study comparing pulmonary function changes over time solely in patients with IPAF showed a continuous decline in FVC from $82 \%$ to $70.5 \%$ at a median follow-up of 45 months [16]. Chartrand et al. [13] reported that adjusted FVC and $\mathrm{DL}_{\mathrm{CO}}$ by age, sex, and history of smoking were stable at 4-year follow-up in patients with IPAF. With regard to pulmonary function, patients with IPAF tend to show worsening of FVC but relatively conserved $\mathrm{DL}_{\mathrm{CO}}$ over time.

Yoshimura et al. [17] reported that patients with IPAF have a higher prevalence of AE events than those with IIP. However, in subgroup analysis comparing AE events between IPAF with a UIP pattern and IIP with IPF, and between IPAF with an NSIP pattern and IIP with NSIP, showed non-significant results [17]. AE events are the leading cause of death in ILD. Therefore, comparison of $\mathrm{AE}$ events between IPAF and non-IPAF groups is crucial in predicting survival [69]. In one study, AE events were observed less frequently in patients with IPAF than in those with IPF (25.9\% vs. $35.4 \%$, respectively) [9]. However, there have been only a few studies regarding the incidence of $\mathrm{AE}$ events in patients with IPAF, and the prevalence rates of AE events in patients with IPAF and IPF remain unclear.

The median survival period is longer in patients with IPAF and ARD-ILD than in those with IPF $[9,12,16]$, and the 5-year survival rate was higher in patients with IPAF than in those with IPF (69.5\% vs. $36.8 \%$, respectively, $p<0.001$ ) [16]. There is no significant difference in survival rate between patients with IPAF with a UIP pattern and those with IPF $[7,9,17,70]$. In one study, patients with IPAF showed better survival than did those with ARD-ILD, including SSc, inflammatory myositis, and RA [13]. Another study demonstrated that patients with ARD-ILD had better survival than those with IPAF [7]. Cox regression analysis showed discrepancies in the impacts of IPAF on mortality. Lim et al. [9] and Yoshimura et al. [17] reported lower HR for the diagnosis of IPAF, whereas Chartrand et al. [13] reported that the form of ILD (IPAF or ARD-ILD) is not a significant predictor of mortality.

The predictive factors of mortality in IPAF are older age, UIP pattern, lower baseline $\mathrm{FVC} / \mathrm{DL}_{\mathrm{CO}}$, history of smoking, and presence of AE events [7-9,11-14,16,17]. Several studies included the morphological form of ILD as UIP compared with non-UIP, or NSIP compared with the NSIP + organizing pneumonia (OP)/OP pattern. In subgroup analyses, some studies showed that patients with IPAF with a UIP pattern had poorer survival than those with IPAF with a non-UIP pattern $[12,70]$. However, another study demonstrated no significant difference in overall survival between patients with IPAF with a UIP pattern and those with IPAF with an NSIP pattern [8]. The UIP pattern showed an increased HR in one study (HR, 3.847; 95\% confidence interval [CI], 1.991 to 7.434) [12], but other studies showed that the UIP pattern is not a significant factor predicting mortality (HR, 1.53; 95\% CI, 0.54 to 4.31$)[8,14,16]$. Oldham et al. [7] reported that the UIP pattern was associated with increased HR for mortality in univariate analysis but that it lost its significance in multivariate analysis. Other studies divided IPAF cases according to the morphological domain included in the 2015 ERS/ATS classification criteria for IPAF (NSIP, NSIP with OP overlap, and OP) and showed 
the opposite results. Ito et al. [11] reported that IPAF with an NSIP pattern had higher HR than NSIP with OP/OP pattern (HR, 4.48; 95\% CI, 1.28 to 15.77). However, Dai et al. [14] reported that the OP pattern had a higher HR than NSIP or NSIP with an OP pattern (HR, 3.385; 95\% CI, 1.017 to 11.261). With regard to specific autoantibodies, patients with IPAF who had SSc-related autoantibodies (anti-topoisomerase antibody, ANA with a nucleolar or centromere pattern, anti-ribonucleoprotein antibody) had poorer prognoses $[11,14]$, suggesting that a specific IPAF subgroup has a distinctive prognosis compared to other forms of IPAF. Taken together, some prognostic factors (age, baseline pulmonary function, and history of smoking) in IPAF were similar to those in IIP, and other factors (morphological patterns and specific autoantibodies) were solely associated with IPAF. Although the 2015 ERS/ATS classification criteria for IPAF were established to define IPAF and conduct studies on IPAF, physicians should consider the individual characteristics of patients with IPAF for prediction of prognosis.

\section{CONCLUSIONS}

There have been a number of IPAF-associated studies since the establishment of the classification criteria for IPAF in 2015. A specific morphological pattern is associated with prognosis, and immunosuppressants are widely used for IPAF. However, most studies were conducted retrospectively. Little is known about IPAF. Considering its complexity and heterogeneity, more well-designed clinical interventional and prospective observational studies would enhance our knowledge of IPAF. Furthermore, the 2015 classification criteria should be revised to facilitate more precise clinical research. In the diagnosis and treatment of IPAF, a multidisciplinary team approach by experts in rheumatology, pulmonology, radiology, pathology, and laboratory medicine is necessary.

\section{Conflict of interest}

No potential conflict of interest relevant to this article was reported.

\section{Acknowledgments}

This research was supported by a grant from the Basic
Science Research Program through the National Research Foundation of Korea, funded by the Ministry of Education, Science and Technology, Republic of Korea (NRF-2018R1DiA1A02050982).

\section{REFERENCES}

1. Park SW, Baek AR, Lee HL, et al. Korean guidelines for diagnosis and management of interstitial lung diseases: part 1. Introduction. Tuberc Respir Dis (Seoul) 2019;82:269-276.

2. Kinder BW, Collard HR, Koth L, et al. Idiopathic nonspecific interstitial pneumonia: lung manifestation of undifferentiated connective tissue disease? Am J Respir Crit Care Med 2007;176:691-697.

3. Fischer A, Antoniou KM, Brown KK, et al. An official European Respiratory Society/American Thoracic Society research statement: interstitial pneumonia with autoimmune features. Eur Respir J 2015;46:976-987.

4. Fischer A, West SG, Swigris JJ, Brown KK, du Bois RM. Connective tissue disease-associated interstitial lung disease: a call for clarification. Chest 2010;138:251-6.

5. Vij R, Noth I, Strek ME. Autoimmune-featured interstitial lung disease: a distinct entity. Chest 2011;140:1292-1299.

6. Corte TJ, Copley SJ, Desai SR, et al. Significance of connective tissue disease features in idiopathic interstitial pneumonia. Eur Respir J 2012;39:661-668.

7. Oldham JM, Adegunsoye A, Valenzi E, et al. Characterisation of patients with interstitial pneumonia with autoimmune features. Eur Respir J 2016;47:1767-1775.

8. Ahmad K, Barba T, Gamondes D, et al. Interstitial pneumonia with autoimmune features: clinical, radiologic, and histological characteristics and outcome in a series of 57 patients. Respir Med 2017;123:56-62.

9. Lim JU, Gil BM, Kang HS, Oh J, Kim YH, Kwon SS. Interstitial pneumonia with autoimmune features show better survival and less exacerbations compared to idiopathic pulmonary fibrosis. BMC Pulm Med 2019;19:120.

10. Sambataro G, Sambataro D, Torrisi SE, et al. Clinical, serological and radiological features of a prospective cohort of Interstitial Pneumonia with Autoimmune Features (IPAF) patients. Respir Med 2019;150:154-160.

11. Ito Y, Arita M, Kumagai S, et al. Serological and morphological prognostic factors in patients with interstitial pneumonia with autoimmune features. BMC Pulm Med 
2017;17:111.

12. Kim HC, Lee JH, Chae EJ, Song JS, Song JW. Long-term clinical course and outcome of interstitial pneumonia with autoimmune features. Respirology 2020;25:636-643.

13. Chartrand S, Lee JS, Swigris JJ, Stanchev L, Fischer A. Clinical characteristics and natural history of autoimmune forms of interstitial lung disease: a single-center experience. Lung 2019;197:709-713.

14. Dai J, Wang L, Yan X, et al. Clinical features, risk factors, and outcomes of patients with interstitial pneumonia with autoimmune features: a population-based study. Clin Rheumatol 2018;37:2125-2132.

15. Chartrand S, Swigris JJ, Stanchev L, Lee JS, Brown KK, Fischer A. Clinical features and natural history of interstitial pneumonia with autoimmune features: a single center experience. Respir Med 2016;119:150-154.

16. Sebastiani M, Cassone G, De Pasquale L, et al. Interstitial pneumonia with autoimmune features: a single center prospective follow-up study. Autoimmun Rev 2020;19:102451.

17. Yoshimura K, Kono M, Enomoto Y, et al. Distinctive characteristics and prognostic significance of interstitial pneumonia with autoimmune features in patients with chronic fibrosing interstitial pneumonia. Respir Med 2018;137:167-175.

18. Tirelli C, Morandi V, Valentini A, et al. Multidisciplinary approach in the early detection of undiagnosed connective tissue diseases in patients with interstitial lung disease: a retrospective cohort study. Front Med (Lausanne) 2020;7:11.

19. Tian M, Huang W, Ren F, et al. Comparative analysis of connective tissue disease-associated interstitial lung disease and interstitial pneumonia with autoimmune features. Clin Rheumatol 2020;39:575-583.

2o. Collins BF, Spiekerman CF, Shaw MA, et al. Idiopathic interstitial pneumonia associated with autoantibodies: a large case series followed over 1 year. Chest 2017;152:103112.

21. Alevizos MK, Giles JT, Patel NM, Bernstein EJ. Risk of progression of interstitial pneumonia with autoimmune features to a systemic autoimmune rheumatic disease. Rheumatology (Oxford) 2020;59:1233-1240.

22. Schurawitzki H, Stiglbauer R, Graninger W, et al. Interstitial lung disease in progressive systemic sclerosis: high-resolution CT versus radiography. Radiology 1990;176:755-759.
23. Tashkin DP, Elashoff R, Clements PJ, et al. Cyclophosphamide versus placebo in scleroderma lung disease. N Engl J Med 2006;354:2655-2666.

24. Sanchez-Cano D, Ortego-Centeno N, Callejas JL, et al. Interstitial lung disease in systemic sclerosis: data from the Spanish scleroderma study group. Rheumatol Int 2018;38:363-374.

25. Fathi M, Dastmalchi M, Rasmussen E, Lundberg IE, Tornling G. Interstitial lung disease, a common manifestation of newly diagnosed polymyositis and dermatomyositis. Ann Rheum Dis 2004;63:297-301.

26. Chen IJ, Jan Wu YJ, Lin CW, et al. Interstitial lung disease in polymyositis and dermatomyositis. Clin Rheumatol 2009;28:639-646.

27. Hayashi S, Tanaka M, Kobayashi H, et al. High-resolution computed tomography characterization of interstitial lung diseases in polymyositis/dermatomyositis. J Rheumatol 2008;35:260-269.

28. Bongartz T, Nannini C, Medina-Velasquez YF, et al. Incidence and mortality of interstitial lung disease in rheumatoid arthritis: a population-based study. Arthritis Rheum 2010;62:1583-1591.

29. Gochuico BR, Avila NA, Chow CK, et al. Progressive preclinical interstitial lung disease in rheumatoid arthritis. Arch Intern Med 2008;168:159-166.

30. Guisado-Vasco P, Silva M, Duarte-Millan MA, et al. Quantitative assessment of interstitial lung disease in Sjögren's syndrome. PLoS One 2019;14:e0224772.

31. Roca F, Dominique S, Schmidt J, et al. Interstitial lung disease in primary Sjögren's syndrome. Autoimmun Rev 2017;16:48-54.

32. Dong X, Zhou J, Guo X, et al. A retrospective analysis of distinguishing features of chest HRCT and clinical manifestation in primary Sjögren's syndrome-related interstitial lung disease in a Chinese population. Clin Rheumatol 2018;37:2981-2988.

33. Damoiseaux J, Andrade LEC, Carballo OG, et al. Clinical relevance of HEp-2 indirect immunofluorescent patterns: the International Consensus on ANA patterns (ICAP) perspective. Ann Rheum Dis 2019;78:879-889.

34. Cottin V, Brown KK. Interstitial lung disease associated with systemic sclerosis (SSc-ILD). Respir Res 2019;20:13.

35. Koenig M, Dieude M, Senecal JL. Predictive value of antinuclear autoantibodies: the lessons of the systemic sclerosis autoantibodies. Autoimmun Rev 2008;7:588-593.

36. Spagnolo P, Cottin V. Genetics of idiopathic pulmonary 
fibrosis: from mechanistic pathways to personalised medicine. J Med Genet 2017;54:93-99.

37. McLean-Tooke A, Moore I, Lake F. Idiopathic and immune-related pulmonary fibrosis: diagnostic and therapeutic challenges. Clin Transl Immunology 2019;8:e1086.

38. Newton CA, Oldham JM, Ley B, et al. Telomere length and genetic variant associations with interstitial lung disease progression and survival. Eur Respir J 2019;53:1801641.

39. Linn-Rasker SP, van der Helm-van Mil AH, van Gaalen FA, et al. Smoking is a risk factor for anti-CCP antibodies only in rheumatoid arthritis patients who carry HLADRB1 shared epitope alleles. Ann Rheum Dis 2006;65:366371.

40. Lee HS, Irigoyen P, Kern M, et al. Interaction between smoking, the shared epitope, and anti-cyclic citrullinated peptide: a mixed picture in three large North American rheumatoid arthritis cohorts. Arthritis Rheum 2007;56:1745-1753.

41. Schiffenbauer A, Faghihi-Kashani S, O'Hanlon TP, et al. The effect of cigarette smoking on the clinical and serological phenotypes of polymyositis and dermatomyositis. Semin Arthritis Rheum 2018;48:504-512.

42. Karabulut G, Kitapcioglu G, Inal V, et al. Cigarette smoking in primary Sjögren's syndrome: positive association only with ANA positivity. Mod Rheumatol 2011;21:602607.

43. Kang J, Jeong SH, Lee K, et al. Exacerbation of symptomatic arthritis by cigarette smoke in experimental arthritis. PLoS One 2020;15:e0230719.

44. Aletaha D, Neogi T, Silman AJ, et al. 2010 Rheumatoid arthritis classification criteria: an American College of Rheumatology/European League Against Rheumatism collaborative initiative. Arthritis Rheum 2010;62:25692581.

45. van den Hoogen F, Khanna D, Fransen J, et al. 2013 Classification criteria for systemic sclerosis: an American College of Rheumatology/European League against Rheumatism collaborative initiative. Arthritis Rheum 2013;65:27372747.

46. Sulli A, Secchi ME, Pizzorni C, Cutolo M. Scoring the nailfold microvascular changes during the capillaroscopic analysis in systemic sclerosis patients. Ann Rheum Dis 2008;67:885-887.

47. Shiboski CH, Shiboski SC, Seror R, et al. 2016 American College of Rheumatology/European League against Rheumatism Classification criteria for primary Sjögren's syndrome: a consensus and data-driven methodology involving three international patient cohorts. Arthritis Rheumatol 2017;69:35-45.

48. Aringer M, Costenbader K, Daikh D, et al. 2019 European League against Rheumatism/American College of Rheumatology classification criteria for systemic lupus erythematosus. Arthritis Rheumatol 2019;71:1400-1412.

49. Dalakas MC. Inflammatory muscle diseases. N Engl J Med 2015;372:1734-1747.

50. Alba MA, Flores-Suarez LF, Henderson AG, et al. Interstital lung disease in ANCA vasculitis. Autoimmun Rev 2017;16:722-729.

51. Castelino FV, Varga J. Interstitial lung disease in connective tissue diseases: evolving concepts of pathogenesis and management. Arthritis Res Ther 2010;12:213.

52. Gao Y, Moua T. Treatment of the connective tissue disease-related interstitial lung diseases: a narrative review. Mayo Clin Proc 2020;95:554-573.

53. Li Y, Zheng Z, Han Q, et al. IPAF should receive early treatment for sharing similar clinical characteristics as CTD-ILD: a report from 273 Chinese patients. Clin Rheumatol 2020;39:3817-3823.

54. Noble PW, Albera C, Bradford WZ, et al. Pirfenidone in patients with idiopathic pulmonary fibrosis (CAPACITY): two randomised trials. Lancet 2011;377:1760-1769.

55. King TE Jr, Bradford WZ, Castro-Bernardini S, et al. A phase 3 trial of pirfenidone in patients with idiopathic pulmonary fibrosis. N Engl J Med 2014;370:2083-2092.

56. Margaritopoulos GA, Trachalaki A, Wells AU, et al. Pirfenidone improves survival in IPF: results from a real-life study. BMC Pulm Med 2018;18:177.

57. Richeldi L, du Bois RM, Raghu G, et al. Efficacy and safety of nintedanib in idiopathic pulmonary fibrosis. N Engl J Med 2014;370:2071-2082.

58. Lancaster L, Crestani B, Hernandez P, et al. Safety and survival data in patients with idiopathic pulmonary fibrosis treated with nintedanib: pooled data from six clinical trials. BMJ Open Respir Res 2019;6:0000397.

59. Acharya N, Sharma SK, Mishra D, Dhooria S, Dhir V, Jain S. Efficacy and safety of pirfenidone in systemic sclerosis-related interstitial lung disease-a randomised controlled trial. Rheumatol Int 2020;40:703-710.

6o. Distler O, Highland KB, Gahlemann M, et al. Nintedanib for systemic sclerosis-associated interstitial lung disease. N Engl J Med 2019;380:2518-2528.

61. Flaherty KR, Brown KK, Wells AU, et al. Design of the PF- 
ILD trial: a double-blind, randomised, placebo-controlled phase III trial of nintedanib in patients with progressive fibrosing interstitial lung disease. BMJ Open Respir Res 2017;4:e000212.

62. Maher TM, Corte TJ, Fischer A, et al. Pirfenidone in patients with unclassifiable progressive fibrosing interstitial lung disease: design of a double-blind, randomised, placebo-controlled phase II trial. BMJ Open Respir Res 2018;5:e000289.

63. Sambataro G, Vancheri A, Torrisi SE, et al. The morphological domain does not affect the rate of progression to defined autoimmune diseases in patients with interstitial pneumonia with autoimmune features. Chest 2020;157:238-242.

64. Bourn R, James JA. Preclinical lupus. Curr Opin Rheumatol 2015;27:433-439.

65. Smolen JS, Aletaha D, Barton A, et al. Rheumatoid arthri- tis. Nat Rev Dis Primers 2018;4:18001.

66. Hyldgaard C, Hilberg O, Pedersen AB, et al. A population-based cohort study of rheumatoid arthritis-associated interstitial lung disease: comorbidity and mortality. Ann Rheum Dis 2017;76:1700-1706.

67. Peelen L, Wells AU, Prijs M, et al. Fibrotic idiopathic interstitial pneumonias: mortality is linked to a decline in gas transfer. Respirology 2010;15:1233-1243.

68. Song JW, Hong SB, Lim CM, Koh Y, Kim DS. Acute exacerbation of idiopathic pulmonary fibrosis: incidence, risk factors and outcome. Eur Respir J 2011;37:356-363.

69. Leuschner G, Behr J. Acute exacerbation in interstitial lung disease. Front Med (Lausanne) 2017;4:176.

70. Kelly BT, Moua T. Overlap of interstitial pneumonia with autoimmune features with undifferentiated connective tissue disease and contribution of UIP to mortality. Respirology 2018;23:600-605. 